FORMATION Formation emploi

Revue française de sciences sociales

139 | Juillet-Septembre 2017

De l'autonomie dans les parcours professionnels

\title{
L'autonomie des patients, un objectif à atteindre ou une condition requise : les pratiques des services d'insertion dans les centres de médecine physique et de réadaptation
}

Patient autonomy, a goal or a necessary condition : practices of social and vocational services in physical medicine and rehabilitation centers

Autonomie von Patienten - Ziel oder Voraussetzung: Methoden der Dienste für Wiedereingliederung in der Physikalischen und Rehabilitativen Medizin La autonomía de los pacientes, un objetivo a alcanzar o una condición requerida : las prácticas de los servicios de inserción en terapia física y de readaptación

Seak-Hy Lo

\section{(2) OpenEdition}

\section{Édition électronique}

URL : http://journals.openedition.org/formationemploi/5140

DOI : 10.4000/formationemploi.5140

ISSN : 2107-0946

Éditeur

La Documentation française

Édition imprimée

Date de publication : 15 octobre 2017

Pagination : 71-89

ISSN : 0759-6340

\section{Référence électronique}

Seak-Hy Lo, «L'autonomie des patients, un objectif à atteindre ou une condition requise : les pratiques des services d'insertion dans les centres de médecine physique et de réadaptation », Formation emploi [En ligne], 139 | Juillet-Septembre 2017, mis en ligne le 15 octobre 2019, consulté le 30 octobre 2020. URL : http://journals.openedition.org/formationemploi/5140; DOI : https://doi.org/10.4000/ formationemploi.5140 


\title{
L'autonomie des patients, un objectif à atteindre ou une condition requise : les pratiques des services d'insertion dans les centres de médecine physique et de réadaptation
}

\author{
SEAK-HY LO \\ Sociologue démographe, Agence régionale de santé Île-de-France
}

Résumé

L'autonomie des patients, un objectif à atteindre ou une condition requise : les pratiques des services d'insertion dans les centres de médecine physique et de réadaptation

Larticle interroge la manière dont les services d'insertion professionnelle, au sein de deux centres hospitaliers, appréhendent la notion d'autonomie dans leurs pratiques d'accompagnement de patients devenus handicapés. L'autonomie est, dans certains cas, un objectif à atteindre ; pour d'autres, une condition requise pour la prise en charge du patient. Selon la posture adoptée, l'accompagnement est soit personnalisé, c'est-à-dire suivant des protocoles prédéfinis qui seront adaptés aux personnes, soit individualisé, imposant aux professionnels d'innover dans leurs pratiques.

Mots clés : travailleur handicapé ; accompagnement professionnel ; réinsertion professionnelle ; établissement hospitalier

Abstract

Patient autonomy, a goal or a necessary condition: practices of social and vocational services in physical medicine and rehabilitation centers

This article aims to present how social and vocational services of two hospitals perceive the notion of autonomy in their work with persons who became disabled. In some cases, autonomy can be seen as a goal and in others, as a necessary condition for the management of the patient. According to the position adopted, the support of the patient is personalized (i.e. following predefined procedures adapted to the patients) or individualized (which supposes innovative practices on the part of professionals).

Keywords: disabled worker ; coaching ; re-integration into the labour market ; hospital Journal of Economic Literature: J 14 ; J 24

Traduction : Auteure. 
L'autonomie est aujourd'hui " une valeur" vers laquelle l'individu doit tendre (Ehrenberg, 2010). Elle est progressivement devenue une norme institutionnelle dans les politiques sociales comme dans les politiques de la santé, dont l'objectif est de donner les capacités à chacun d'accéder à ses choix. À partir des années 1980, "l'objectif affiché est de passer d'une culture de la 'dépendance' à une culture de la 'participation', de la 'responsabilisation' et de l' 'activation'” (Bacqué \& Biewerner, 2013, p. 132).

Les discours sur la responsabilisation des individus se généralisent, incitant la personne à être active afin de devenir autonome dans son parcours (Astier, 2007). Cette notion d'autonomie, qui est utilisée de manière différente selon les champs, renvoie à une acception commune de liberté et d'indépendance où l'individu est acteur, capable de décision.

Cette nouvelle approche dans les politiques sociales ou de la santé rend nécessaire de faire évoluer les dispositifs afin de donner les moyens aux individus de s'autonomiser. Mais cela soulève la question de la capacité du nouvel État social actif (Vielle, Pochet, \& Cassiers, 2006) à fournir les supports (Castel \& Haroche, 2001) ainsi que les ressources (Sen, 1987) aux individus vulnérables pour leur permettre de développer leur projet.

Ainsi, de nouveaux modes de prise en charge centrés sur l'individu ont émergé cette dernière décennie. Il est attendu à présent de l'individu qu'il soit auteur de son parcours, contrairement à la vision de la personne passive, dépendante du système de protection sociale qui l'isole socialement (Castra, 2003). Le travail social n'est plus un travail sur autrui, mais un travail avec la personne. D'ailleurs, il n'est plus question de "prise en charge », mais d' "accompagnement » des personnes vers leur insertion sociale et professionnelle (Paul, 2007).

Au sein de la médecine physique et de réadaptation (MPR), cette vision de la prise en charge centrée sur l'individu est partagée. Cette spécialité médicale vise la rééducation des personnes ayant des atteintes fonctionnelles. Cette prise en compte de l'individu n'est pas récente dans la MPR, dont l'objectif est aussi la réinsertion sociale des personnes handicapées. En effet, depuis les années 1950, le travail a une très forte valeur sociale par ses fonctions d'intégration, de reconnaissance et d'utilité sociale (Ehrenberg, 2011 ; Méda, 1995), amenant à l'idée d'une réinsertion des personnes handicapées par la réadaptation et le retour au travail. Bien qu'il soit souvent problématique pour les personnes ayant des contraintes de santé, le retour en emploi n'est que peu abordé dans les services hospitaliers français, la question étant renvoyée à l'extérieur du monde sanitaire. Pourtant, à partir des années 1990, plusieurs centres de MPR se sont dotés d'un service d'insertion professionnelle. C'est notamment le cas des centres Laville et Lamer, au sein desquels la recherche a été réalisée. Ce sont de grands établissements de santé, spécialisés dans la MPR, et dont l'histoire est liée à celle des politiques de réadaptation, en France (Frattini, 2008). Tous deux ont une grande capacité d'accueil (plus de 150 places en MPR adultes) et bénéficient d'une forte reconnaissance dans le secteur, notamment dans le domaine des pathologies 
traumatiques et neurologiques (amputation, paraplégie, hémiplégie, etc.). Dans chacun de ces centres, un service d'insertion professionnelle déploie aujourd'hui son activité et a adopté la démarche d'insertion précoce (DPI). Elle envisage la réinsertion professionnelle alors que le patient est hospitalisé afin de lui donner les meilleures chances de retourner en emploi. Le décret du 17 avril 2008, qui inscrit l'insertion sociale et professionnelle comme un objectif de travail pour les services de MPR, nous amène à questionner la place de ce patient-acteur pendant son parcours hospitalier, mais aussi l'intégration d'un tel objectif dans le champ sanitaire. Les centres de MPR se doivent de redonner un maximum d'autonomie à la personne dans sa vie " habituelle ", l'autonomie étant conçue en opposition à un état de dépendance.

L'article interroge la manière dont les services d'insertion professionnelle, au sein de groupes hospitaliers, appréhendent cette notion d'autonomie dans les parcours de patients devenus handicapés. Ces patients ont une déficience motrice ou neuro-motrice (tétraplégie, paraplégie, hémiplégie, amputation, lombalgie grave, dorsalgie grave, etc.), causée par un accident ou une maladie.

Ainsi, à partir des pratiques des professionnels de deux services d'insertion, nous analysons leurs principes de travail et leur mise en ouvre. Puis, à travers deux parcours-types, nous montrons comment cette notion d'autonomie est appréhendée dans les accompagnements vers la réinsertion professionnelle.

\section{Encadré 1 : Point de méthode}

Au sein des services d'insertion professionnelle Lamer et Laville, deux services contrastés implantés dans des régions différentes, et avec une organisation et une histoire distinctes, les terrains ethnographiques ont été réalisés de janvier 2010 à avril 2012. Ces services illustrent bien les pratiques d'accompagnement des patients utilisant les dispositifs sanitaires, sociaux et médico-sociaux.

Nous y avons observé des réunions de travail, des rendez-vous et échanges avec les patients, des conversations entre membres de l'équipe, auxquels s'ajoute la participation à des événements particuliers, comme des visites d'entreprises, ayant lieu avant la reprise du travail du patient.

Nous avons également réalisé des entretiens en plus des discussions informelles avec des professionnels. Ces derniers ont fourni l'accès à leurs dossiers de travail qui comportaient des notes sur le patient, des comptes rendus de rencontres, de visites d'entreprises ou encore d'aménagements réalisés, des échanges de courriels et des copies des dossiers de demandes d'aide sociale. Ils offraient une grande richesse d'informations pour comprendre le travail au long cours des professionnels.

Les entretiens formels visaient à expliciter les pratiques d'accompagnement à partir des expériences auprès des patients rencontrés dans le second volet de l'enquête. Au total, dans le service Lamer, quatre chargés d'insertion ont été interrogés et trois membres de l'équipe dans le service Laville. Ils ont été interrogés d'une à trois fois (12 entretiens au total). 
Parallèlement, un second volet a été mené auprès de personnes souffrant d'une déficience motrice ou neuro-motrice et ayant été hospitalisées dans un des deux centres étudiés. La sélection des enquêtés s'est opérée grâce au concours des centres hospitaliers qui ont proposé des personnes répondant à nos critères d'enquête : personnes âgées de 20 à 59 ans, toutes en emploi au moment de la survenue de la déficience et qui sont devenues handicapées à l'âge adulte. Sont recueillis les récits de vingt-quatre personnes. Le premier entretien s'apparente à un entretien biographique (Bertaux, 1997 ; Demazière \& Dubar, 1997) retraçant le parcours professionnel et de soins. Puis, au cours des entretiens suivants, nous avons questionné les évolutions des situations. Au total, une centaine d'entretiens a été recueillie et analysée pour ce volet.

Pour l'analyse, nous avons adopté une approche compréhensive et comparative des éléments recueillis. A partir de l'ensemble des données, nous avons cherché à restituer les logiques organisationnelles des deux services. Puis, nous nous sommes appuyés sur des « configurations » de travail à partir de leur ressemblances et dissemblances pour comprendre les pratiques professionnelles (Becker, 2002). Pour cela, nous avons croisé différentes sources d'informations : les fascicules de présentation du service recueillis sur les terrains, les entretiens, les documents de travail, les rapports d'activité, les observations, etc. Ces données ont permis de caractériser les services en distinguant leur histoire et leur fonctionnement. Pour l'analyse des parcours, nous avons également utilisé le contenu des documents de travail des professionnels pour mettre en perspective la chronologie des faits énoncés par les interviewés. Cela nous a également renseigné sur des éléments que les enquêtés ont tus dans leur récit, les "zones blanches » (Bertaux, 1997), considérées comme moins importantes. En croisant cette subjectivité à d'autres faits et à d'autres récits, nous enrichissons notre registre de compréhension.

\section{Les principes de travail : protéger, considérer le contexte, favoriser l'autonomie du patient}

Les services d'insertion au sein des centres hospitaliers Lamer et Laville ont pour missions d'accompagner les personnes hospitalisées suite à un accident ou à une phase aigüe de leur maladie, afin de les aider dans leur insertion professionnelle. La première rencontre avec les services d'insertion a lieu au cours de l'hospitalisation ou à la fin de leur séjour au centre.

Les personnes qui travaillent au sein de ces services que nous appelons, dans cet article, " les professionnels », s'appuient sur trois grands principes qui régissent leur accompagnement : protéger le patient, prendre en compte son contexte dans leur évaluation et lui donner les moyens d'être autonome.

\subsection{Protéger le patient : un souci constant}

La protection du patient est présentée comme un souci constant pour les professionnels, et cela sur trois dimensions particulières : la santé, les droits sociaux et le devenir professionnel : "Ne pas le [le patient] mettre en danger sur quelque ligne que ce soit, aussi bien 
sur le plan de la santé, sur le plan juridique, que par rapport à l'employeur. Moi, c'est ce sur quoi je suis vigilante» (Mme Z, médecin responsable, service Laville).

Ainsi, l'évaluation des situations tient tout d'abord compte de l'évolution de l'état de santé. La question est de savoir à quel moment du parcours de soin il est judicieux d'envisager avec le patient la reprise du travail. De manière consensuelle, les professionnels reconnaissent que cette étape doit respecter un certain ordre. La priorité va à la récupération des activités primaires : se lever, se déplacer, boire, manger, s'habiller. Puis, à celles de la "vie quotidienne ", comme sortir, faire à manger, mais aussi mieux maîtriser certaines aides techniques, préparer le retour au domicile, passer ou repasser le permis de conduire. Ces acquis conditionnent la reprise d'une activité professionnelle qui est rarement la préoccupation centrale du patient durant l'hospitalisation. Il faut laisser du temps au patient, ne pas brûler les étapes. La première rencontre avec l'équipe d'insertion est le moyen de vérifier que les conditions pour entamer la réadaptation professionnelle sont réunies.

Lévolution de la santé du patient peut les conduire à avancer, freiner ou repousser l'accompagnement. Ils n'hésitent pas à ajourner le projet, notamment lorsqu'ils estiment la réadaptation fonctionnelle du patient encore fragile. Reprendre le travail trop tôt expose aussi l'individu à des difficultés professionnelles. En cas d'échec, il peut, d'une part, rencontrer des difficultés à se réinvestir dans un nouveau projet et, d'autre part, ne plus être crédible auprès de son employeur ou son réseau professionnel.

Dans tous les cas, il faut garantir a minima les droits sociaux qui constituent l'assurance d'une ressource financière, en cas d'échec du projet. Le travail garantit des droits considérés comme plus protecteurs (indemnités journalières, pension d'invalidité, etc.) que ceux de l'action sociale (l'Allocation adulte handicapée).

Les professionnels examinent l'ensemble des possibilités offertes. Ils balisent ainsi le projet, n'hésitant pas à tirer le maximum des dispositifs. Par exemple, il n'est pas rare que les trois années règlementaires d'arrêt maladie soient utilisées jusqu'au dernier jour pour construire le projet et consolider l'ensemble des aspects avant le retour en emploi. Le temps de l'hospitalisation est considéré comme un moment pour mettre en place le projet : "L'objectif est d'anticiper les choses pendant l'hospitalisation ou de faire les choses alors que vous êtes encore à l'hôpital» (Mme Z, médecin responsable, service Laville).

C'est ainsi que, par souci de réalisme par rapport au marché du travail et à l'insertion des personnes handicapées, le maintien en emploi est une des actions privilégiées des services.

Dans la mesure de leurs compétences, ils aident le patient dans différentes situations : le licenciement, la reconversion, la recherche d'emploi ou encore la sortie de l'emploi avec une procédure de mise en invalidité. Ainsi, ils ne le laissent pas seul face à des procédures qui peuvent vite devenir complexes au vu de l'organisation des dispositifs liés au handicap. 


\subsection{Considérer le patient dans son contexte à la fois médical et professionnel}

De ce premier principe de protection découle un second principe, qui est un héritage du mode opératoire de la médecine physique et de la réadaptation : prendre le patient dans son contexte et non pas uniquement en fonction d'une évaluation de son état physiologique.

Les professionnels analysent, par exemple, les possibilités de reprise du travail dans une double perspective qui tient compte des capacités fonctionnelles - évaluées non pas en fonction d'une norme " médicale " standardisée, mais selon le travail envisagé -, et du contexte professionnel, en particulier de la situation du marché du travail. Cette manière d'appréhender le handicap n'est pas sans rappeler la notion de "situation de handicap " créée par l'interaction entre un individu, ses caractéristiques personnelles et des facteurs environnementaux (Ravaud, 1999).

S’agissant des capacités fonctionnelles du patient, trois dimensions sont régulièrement interrogées : la nature des tâches (le patient peut-il accomplir les tâches liées à son travail ?), la durée (le patient peut-il réaliser ces tâches dans la durée sans répercussion sur sa santé ?) et la quantité (le patient peut-il réaliser toutes les tâches demandées ?). La première dimension conditionne la reprise de l'activité professionnelle antérieure ou un éventuel reclassement dans l'entreprise. Les deux autres dimensions orientent vers une adaptation du poste de travail.

Quoi qu'il en soit, les solutions les plus proches de la situation antérieure à l'accident sont privilégiées. Elles sont en effet considérées comme ayant plus de chance d'aboutir dans des délais raisonnables, comme l'illustre le cas d'un patient atteint d'une grave lombalgie, imprimeur de métier et cadre dans une entreprise souhaitant le reprendre. Son statut de cadre est perçu comme sécurisé et socialement valorisé ; l'entreprise est implantée dans plusieurs régions, ce qui offre de bonnes possibilités de reclassement. Les professionnels, considérant ces différents facteurs comme favorables, encouragent le maintien au travail de cette personne, malgré ses problèmes de santé. A contrario, la situation d'un artisan ayant une lombalgie similaire, devant porter des charges, mais sans possibilité de reclassement, est considérée comme nettement moins favorable. Le patient sera alors dirigé vers une reconversion. Le cadre est jugé employable car ses conditions d'emploi sont adaptables à ses capacités physiques. Il est encouragé à reprendre son travail en adaptant ce dernier à ses nouvelles contraintes. L'environnement et le statut professionnels sont ainsi essentiels dans l'évaluation des capacités de travail.

\subsection{Favoriser l'autonomie du patient dans la démarche d'insertion}

Les professionnels promeuvent l'autonomie d'action : le patient doit être acteur de son parcours. Ainsi, le projet de vie "incluant obligatoirement une dimension professionnelle " 
doit se construire avec la "participation volontaire et active " ${ }^{1}$ de la personne. Il s'agit donc de la guider en lui indiquant la procédure à suivre, les sources d'informations, ainsi que les ressources utiles : "Je vous donne les éléments pour aller rechercher les informations qui vous intéressent pour un futur métier : allez à tel endroit, prenez les fiches ONISEP (Office national d'information sur les enseignements et les professions), etc. " (Mme V, chargée d'insertion, service Laville).

S'ils estiment que le patient a une bonne compréhension de la situation et témoigne d'une certaine assurance, ils l'encouragent à entreprendre lui-même les démarches, les chargés d'insertion n'intervenant qu'en soutien. En proposant au patient certaines ressources, le professionnel lui enseigne des procédés qu'il pourra remobilisés. Cette pratique s'apparente à une éducation du patient qui, d'une part, lui donne le pouvoir d'agir et, d'autre part, lui permet d'être responsable de ses choix et de son parcours, laissant supposer qu'il est ou devient autonome (Jaeger, 2009)

Les intervenants prennent toutefois l'initiative d'intervenir directement lorsque les tâches sont complexes, ingrates ou susceptibles de mettre le patient en difficulté. En effet, les dossiers de demandes d'aide financière étant nombreux, il n'est pas rare de voir les chargés d'insertion les compléter avec ou sans le patient. Ils n'hésitent pas à appeler les différents organismes directement pour prendre le relais des démarches administratives qui peuvent épuiser et décourager le patient.

Les professionnels ont un rôle de soutien, à la fois technique et psychologique, à différents niveaux, ce qui exige qu'ils soient polyvalents et mobilisés. Assurer ce relais est une manière de maintenir le patient dans le projet et d'en garantir la continuité en cas de défaillance passagère.

L'autonomie est donc perçue comme une implication du patient dans un projet d'insertion dans lequel il s'investit plus ou moins intensément, selon le contexte, les étapes et ses capacités. Toutes les actions produites par le patient sont des preuves de son engagement dans la démarche. Cependant, il n'est pas attendu que le patient agisse seul.

Dans cette première partie, nous avons exposé les trois grands principes (protéger le patient, le considérer dans son environnement et favoriser son autonomie) qui régissent le travail des professionnels des services d'insertion. Ces principes, qui doivent être présents à chaque étape de l'accompagnement, révèlent déjà la complexité du positionnement de ces professionnels : ils oscillent entre la mise en action, l'autonomie, et la protection du patient, tout en tenant compte du contexte évolutif de ces personnes. Ces principes sont mis en avant successivement selon les étapes de l'accompagnement, comme nous le verrons dans la suite.

1. Extrait du site internet promouvant la démarche précoce d'insertion (DPI) : http://www.cometefrance. org/print.php?page_id=101 - actif en septembre 2017. 


\section{L'accompagnement : entre démarche classique et pratique innovante}

Dans cette partie, à travers deux parcours types, nous montrons les pratiques des professionnels et leurs manières d'appréhender concrètement un patient " autonome ".

Le premier parcours, celui d'Adrien, révèle la manière dont les professionnels conçoivent un accompagnement "classique ", avec des étapes à suivre, des contraintes auxquelles il faut être attentif et comment ce travail d'accompagnement s'insère dans un champ d'intervention plus ou moins balisé. Le second parcours, celui de Rémi, expose quant à lui un accompagnement plus inédit qui mobilise les équipes et les amène à innover.

\subsection{Un accompagnement « classique » : le parcours d'Adrien}

Pour illustrer les accompagnements que les professionnels considèrent comme « classiques ", nous prenons le cas d'Adrien, 24 ans, informaticien en contrat à durée déterminée (CDD) dans un institut public qu'il devait à terme intégrer en contrat à durée indéterminée (CDI). Il a été victime d'un accident sur le trajet de son travail. Le jeune homme est devenu paraplégique et a été suivi pendant plus d'un an dans un centre de MPR

Les méthodes de travail des professionnels sont ainsi exposées dans la suite : rechercher un état stable au patient, enrôler des personnes ressources, se doter de bons outils et tenir un agenda aux multiples contraintes.

\subsubsection{Rechercher un état « stable » comme préalable}

Le service d'insertion rencontre Adrien pour la première fois pendant son hospitalisation, lorsque celui-ci s'interroge sur son retour au travail. Il a conservé des liens avec ses supérieurs hiérarchiques qui souhaitent son retour, d'autant plus que l'institut mène une politique en faveur des travailleurs handicapés. À la première rencontre, les professionnels posent une série de questions standards afin de comprendre sa situation et son projet. Adrien est interrogé sur sa santé et l'évolution de la réadaptation, mais aussi sur son parcours scolaire, sa vie professionnelle, familiale et relationnelle. Ce n'est qu'au terme d'une évaluation précise des capacités fonctionnelles, de l'environnement et des dispositions professionnelles que le service juge de la suite possible du projet. Puis, systématiquement, à chaque rencontre, les professionnels questionnent le patient sur les trois dimensions de la santé, car celle-ci va rythmer en grande partie l'accompagnement du patient : "Quand on sait que ce n'est pas stable, on ne va pas travailler sur tout et n'importe quoi, n'importe quand, au détriment de sa santé» (Mme V, chargée d'insertion, service Laville).

Dans le cas d'Adrien, la chargée d'insertion a remarqué des troubles de l'attention qui l'inquiètent pour une reprise du travail. Les professionnels façonnent ainsi un nouveau 
circuit de soins en l'orientant vers un bilan neuro-psychologique. Il s'agit d' " améliorer » ses capacités avant d'entreprendre une réinsertion professionnelle.

Parallèlement, le service d'insertion incite Adrien à résoudre une série de difficultés liées à l'accessibilité de son domicile, des lieux de soin, de son lieu de travail et des transports. Ainsi, après avoir repassé son permis et acheté une voiture - ses premiers objectifs fixés par le service d'insertion - celui-ci continue à le guider vers les étapes suivantes : "Je dois trouver un appartement, puis je me donne deux mois pour m'habituer à l'environnement de cet appartement, savoir où trouver les commerces à proximité, trouver une tierce personne, organiser ma vie personnelle. A l'issue des deux mois, je commence le travail petit à petit. Je commence par un mi-temps thérapeutique pour voir la fatigabilité, m’organiser parce que j'ai des soins à faire tous les jours [...]. Comme là, je ne trouve pas encore d'appartement, le délai commence petit à petit à séloigner " (Adrien, 24 ans, informaticien, paraplégique).

Sur les conseils du service d'insertion, le jeune homme retarde sa reprise du travail pour optimiser ses conditions de retour.

La stabilité n'est donc pas considérée dans l'absolu, mais dans un contexte donné, et elle est évolutive selon les étapes. De cette manière, le service adapte l'accompagnement en fonction des capacités et de l'environnement du patient.

\subsubsection{Enrôler des personnes ressources}

Pour avancer dans le projet, les professionnels se mettent en quête d'alliés au sein de l'établissement employeur ou dans les organismes gérant des droits sociaux.

Les professionnels incitent les patients à garder le lien avec leur entreprise pendant et après l'hospitalisation. Ainsi, Adrien assure la médiation avec le service d'insertion qui n'a pas de légitimité pour entrer dans son institut.

Dans les entreprises, les professionnels cherchent toujours des interlocuteurs - des alliés - sur lesquels ils peuvent se reposer et avec lesquels ils pourront entreprendre des actions : "L'objectif est de trouver un allié qui fasse l'intermédiaire pour les jours où je n'y suis pas» (Mme V, chargée d'insertion, service Laville).

Parmi ces interlocuteurs, le médecin du travail représente une ressource importante. Lorsque le médecin du travail n'est pas partenaire dans le processus, comme dans le cas d'Adrien, les professionnels cherchent à atteindre d'autres personnes, telles que l'assistant social, le représentant de la Mission handicap ou des Ressources humaines de l'entreprise. Ces personnes constituent des ressources pour sécuriser le maintien en emploi. Ils sont en mesure d'intervenir pour adapter l'environnement, ou encore assurer une fluidité des interactions.

Les chargés d'insertion se positionnent dans la démarche d'insertion comme des défenseurs des intérêts des patients, en opposition à un monde du travail qui ne facilite pas l'emploi des personnes handicapées. Les travailleurs handicapés ont des droits spéci- 
fiques, mais pour les faire valoir, il faut des informateurs au sein de l'entreprise qui connaissent le fonctionnement et les procédures de celle-ci, auxquels le patient n'a pas toujours accès. Lorsque la collaboration est établie, ils peuvent devenir des porte-paroles du service et plaider en faveur du patient (Astier, 2010). De ce fait, ils constituent une importante ressource mobilisable.

Ce mécanisme d'enrôlement des " alliés " s'apparente à celui décrit par Callon et Latour, c'est-à-dire un "mécanisme par lequel un rôle est défini et attribué à un acteur qui l'accepte. L'enrôlement est un intéressement réussi"(Callon, 1986, p. 189). Celui-ci permet de constituer un tissu social solide qui est pour le patient un support, sans lequel l'action du service d'insertion ne pourrait se mettre en place. Ce travail commun crée par la suite une nouvelle ramification au sein du réseau de partenaires.

\subsubsection{Se doter des bons outils}

Afin d'accompagner au mieux le patient, les professionnels recourent, selon leurs propres termes, à des " outils» de nature différente : techniques (véhicule adapté...), cognitifs ${ }^{2}$, juridiques ${ }^{3}$ et partenariaux (avec la Caisse primaire d'assurance maladie, l'Association de gestion du fonds pour l'insertion des personnes handicapées - Agefiph,...). Lors des prises en charge, ils expérimentent des combinaisons astucieuses de ces outils, susceptibles d'augmenter leurs marges de manœuvre. Dans le cas d'Adrien, les professionnels en ont utilisé différents types, selon leur protocole de travail, pour lui permettre d'être le plus autonome possible. Pour cela, ils lui proposent leur expertise dans l'adaptation de son logement, une évaluation de la conduite sur un véhicule adapté avec l'auto-école partenaire, leurs connaissances en matière d'aménagement du domicile et lieu de travail. Parallèlement, ils l'encouragent à utiliser les différents dispositifs médico-sociaux existants pour faire valoir ses droits en tant que personne handicapée. Ainsi, ils le mettent en lien avec la Maison départementale pour les personnes handicapées (MDPH), afin qu'il soit reconnu comme travailleur handicapé, mais aussi qu'il bénéficie des différentes aides financières pour l'adaptation de son logement, une aide-ménagère, etc.

Pour appuyer le dossier de demande, ils étayent le dossier médical en précisant les difficultés qu'Adrien rencontre dans son quotidien. La position de ces professionnels au sein d'un centre reconnu régionalement et l'expertise qu'ils détiennent dans le domaine $\mathrm{du}$ handicap donnent du poids à leur argumentaire. L'usage des outils juridiques, et des configurations administratives qui en découlent, requiert une bonne connaissance des procédures et du droit que les professionnels acquièrent au fil de leurs accompagnements. La réussite des projets tient souvent à des assemblages complexes d'outils différents qui, avec l'expérience, deviendront des procédures plus ou moins routinisées, notamment pour le maintien en emploi.

2. Expertise médicale, ergonomique, juridique, etc.

3. Le temps partiel thérapeutique, le contrat de réinsertion professionnelle sous indemnité journalière, etc. 
Ces instruments (protocoles construits par les équipes, connaissances techniques, dispositifs sociaux et médico-sociaux, lois, etc.) sont autant de moyens susceptibles de faciliter l'accompagnement pour redonner aux patients un maximum d'autonomie dans leur quotidien, mais également favoriser la réussite de la réinsertion professionnelle. Ils constituent aussi des moyens pour les professionnels de matérialiser le projet par des demandes, des arguments, des descriptions précises de situations, des bilans, qui ancrent le patient dans la réalité décrite par les outils. Le patient est ainsi doté progressivement de plus en plus de moyens d'agir qu'il saisira, ou non, par la suite, selon ses capacités et ses intentions.

L'accumulation de ces outils est un révélateur de la technicité et de l'expertise de ces services d'insertion (Lascoumes, Simard, 2011). Elle leur permet de se légitimer dans leur rôle auprès des patients, mais aussi auprès des partenaires et organisations extérieures. Cependant, la diversité des outils caractérise une hétérogénéité de l'action qui illustre la complexité des situations et de l'accompagnement des patients.

\subsubsection{Tenir un agenda aux multiples contraintes}

Dans le projet de réinsertion professionnelle, il est nécessaire de tenir compte, en plus de la santé du patient, de différents éléments, comme l'aspect financier, l'insuffisance de revenus pouvant être un motif d'abandon. La mise en œuvre d'un projet professionnel ne doit engager des pertes financières qu'a minima. Tous les moyens pour obtenir des ressources sont mobilisés, en faisant appel aux différents droits du salarié : droit du travail, droit de la sécurité sociale et droit de l'action sociale (aide au transport, à la formation pour personne handicapée, etc.). Or, bénéficier des droits sociaux suppose le respect de procédures administratives aux calendriers précis. Les procédures sont appliquées dans des délais déterminés, parfois très contraignants et rigides. Les professionnels tiennent - à cette fin notamment - un véritable agenda du parcours d'insertion. Cette connaissance permet de prévoir l'enchaînement des événements à venir.

Pour Adrien, les professionnels ont d'abord temporisé son retour au travail en intercalant plusieurs étapes qu'ils pensaient nécessaires (un logement adapté, trouver des aides à domicile, etc.). Tous ces éléments qui interviennent dans le projet de retour au travail ont une temporalité qu'il ne faut pas négliger.

Parallèlement, les professionnels veillent à ce que le patient suive les procédures qui lui sont les plus favorables, quitte à le convaincre de ne pas engager d'actions qui pourraient le conduire à un statut moins avantageux.

Dans le cas d'Adrien, malgré un retour au travail préparé et attendu, la réinsertion professionnelle n'est pas à la hauteur de ses attentes : les missions confiées sont soit trop simples, soit trop complexes. Rares sont les collègues disponibles et son supérieur hiérarchique est peu présent. Il se trouve très isolé et doute de ses capacités professionnelles. Au bout de quelques mois, il veut démissionner, mais discute auparavant avec 
la chargée d'insertion. Très vite, elle le dissuade de le faire et lui propose d'abord de se mettre en arrêt maladie pour y réfléchir, car la démission exclut le patient du champ assurantiel du droit du travail et le fait basculer dans le champ assistantiel : "Je lui ai dit qu'il ne fallait pas démissionner. Je voulais qu'il se protège un minimum sur le plan social » (Mme V, chargée d'insertion, service Laville).

Par expérience, les professionnels tendent à freiner toute évolution dans le statut d'emploi, surtout lorsque le patient ne sait pas ce qu'il veut faire par la suite. Ils veulent garder un maximum de possibilités afin que le patient, le temps venu, puisse entreprendre les projets avec le moins de contraintes possibles.

Les procédures liées aux droits sociaux entraînent la mise à l'agenda de questions dans le parcours du patient (Hassenteufel, 2010), posées parfois de manière précoce. Elle conduit les professionnels, mais surtout le patient, à devoir faire des choix dans une temporalité qui n'est pas forcément en adéquation avec celle de son projet ou de son état de santé. Le patient doit en effet prendre des décisions dont il ne connaît ou ne comprend pas toujours les enjeux, ni les conséquences. Ainsi, les règles institutionnelles imposent un calendrier qui restreint sa liberté d'action et de décision.

Au final, les professionnels appréhendent le projet d'insertion professionnelle selon des étapes qui constituent un protocole de travail. Celui-ci est reproduit d'un patient à un autre et est adapté à l'état de santé et à la situation professionnelle de la personne. Le protocole préexiste au projet, qui constitue un cadre semblable dans les deux centres pour tous les patients.

À l'intérieur de ce cadre, des actions plus flexibles seront engagées, selon la volonté du patient et sa situation en termes de capacités et d'environnement de travail. Le patient est considéré comme acteur, essentiellement parce qu'il accepte le projet d'insertion proposé. Cet engagement est toutefois indispensable. Il s'agit d'un contrat moral avec le service d'insertion, mais aussi d'un préalable à l'accès aux réseaux professionnels et sociaux du patient. Dans cette démarche, les professionnels adaptent des outils standardisés existants ou des protocoles en place. Ici, l'accompagnement suit des étapes et utilise une palette d'outils déjà expérimentés, qui peuvent être modulés selon la personne. De ce fait, nous pouvons parler d'une personnalisation de l'accompagnement. Cependant, certains projets ne peuvent être encadrés par le protocole, incitant alors les acteurs à innover.

\subsection{Le patient, moteur dans le renouvellement des pratiques et réseaux des professionnels}

Lorsque la situation d'un patient est plus originale ou lorsque ce dernier se montre très convaincant dans la défense d'un projet inédit, les professionnels n'hésitent pas à s'investir 
dans l'innovation de nouveaux outils. Ce fut notamment le cas de Rémi, chauffeur poidslourd, décidé à reprendre son métier avec le soutien de son employeur, malgré une hémiplégie. Ce patient de 40 ans travaille depuis plus de dix ans dans la même entreprise et aime particulièrement son métier. Il a gardé des liens étroits avec son employeur et ses collègues. Pendant son passage au centre Lamer, Rémi rencontre le service d'insertion avec qui il discute de ses projets.

\subsubsection{Le travail biographique : un temps d'arrêt à l'accompagnement}

Après les premières rencontres avec Rémi, les professionnels estiment que le patient n'est pas prêt à s'engager dans un projet de retour au travail, et n'insistent pas dans ce sens.

En effet, les conséquences du handicap ne sont pas seulement fonctionnelles, elles peuvent toucher à la conception de soi. Des travaux ont montré les difficultés rencontrées par des personnes pour se reconstruire après un accident ou la survenue d'une maladie chronique (Bury, 1982 ; Strauss, 1992 ; Ville, 2005). Le temps du travail biographique est long et variable selon les personnes. Il est donc difficile à maîtriser pour les professionnels. Ainsi, lorsque le patient ne semble pas prêt à s'investir dans une reprise, ils tentent quelques relances et abandonnent parfois : "[La chargée d'insertion] mavait convoqué pour savoir qu'est-ce que je faisais. Je ne sais même plus trop, je l'avais vue une fois ou deux, juste avant de partir de Lamer [...] parce que vous savez, quand vous ne remarchez pas, je sais pas si vous pensez à travailler. Je crois pas. Non, c'est au fur et à mesure du temps que ça vient. " (Rémi, 40 ans, Chauffeur de véhicule poids-lourd, hémiplégie)

Pour Rémi, son transfert vers un autre centre de réadaptation a coupé court à l'accompagnement, surtout que celui-ci était en suspens car sa rééducation n’était pas terminée. Les professionnels sont prudents quant aux conséquences de leurs actions sur le parcours du patient. La dimension sanitaire l'emporte sur la réadaptation sociale. Cette dimension protectrice des services d'insertion est non seulement inscrite dans les pratiques sanitaires, mais elle est également actée dans le champ médico-social, comme le montrent des travaux sur les MPDH (Bureau \& Rist, 2013).

Ainsi, après son retour à domicile, n’ayant pas retrouvé ses capacités physiques, il ne pense pas pouvoir conduire à nouveau un camion poids-lourd. Il essaie alors de s'occuper des tâches ménagères pour participer à la vie familiale, ce qui le satisfait peu.

Malgré l'existence de dispositifs pour favoriser l'insertion professionnelle, certains patients comme Rémi, qui a pourtant rencontré le service d'insertion, ne sont pas toujours orientés vers l'emploi. Il faut parfois attendre que des événements fortuits interviennent dans le parcours pour réactiver le processus.

\subsubsection{Les événements contingents dans le parcours}

Rémi est arrêté depuis bientôt trois ans, moment fatidique qui marque la fin de l'arrêt de travail. Un matin, il lit dans son journal le témoignage d'un chauffeur de camion 
poids-lourd qui a repris son travail malgré une hémiplégie. Il repère très vite le service d'insertion qu'il a rencontré durant sa rééducation. Il s'identifie alors à ce chauffeur et envisage de nouveau une reprise du travail : "Quand j'ai vu dans le journal qu'il y avait une personne qui conduisait un poids-lourd en ayant un plexus, je me suis dit : pourquoi pas moi. Donc c'est pour ça que j’avais appelé Mme B en lui disant... Comme c'était mon métier avant... Parce que j'ai fait ça pendant 11 ans. [...] J'aurais aimé recommencer le travail que je faisais avant. " (Rémi, 40 ans, Chauffeur de véhicule poids-lourd, hémiplégie)

Parallèlement, comme la fin de l'arrêt de travail approche, une assistante sociale de la sécurité sociale le contacte pour faire un bilan et envisager avec lui une mise en invalidité. Rémi profite alors de cet échange pour discuter d'un retour en emploi en citant l'article de presse. C'est alors qu'est envisagé pour lui un projet de réinsertion. L'assistante sociale appelle rapidement le service d'insertion Lamer. De cette manière, Rémi retourne dans le dispositif d'insertion du centre hospitalier grâce à l'aide d'un partenaire extérieur.

Bien que résultant de mesures existantes (politique de communication du service d'insertion et fin programmée de l'arrêt de travail pour longue durée), les dispositifs de réinsertion ne sont parfois activés que grâce à la combinaison inattendue d'événements.

\subsubsection{Quand la motivation est un signe d'autonomie}

Malgré un projet professionnel bien défini, le retour en emploi n'est pas pour autant aisé. Le service d'insertion se doit donc de vérifier avec le patient le réalisme de son projet, mais aussi de son engagement. Ce n'est qu'à ces conditions que les professionnels s'investissent en retour en créant les conditions de sa réalisation. Pour cela, le patient doit satisfaire certains prérequis déjà cités précédemment : un état de santé stabilisé, un soutien social, professionnel et financier fiable et une volonté marquée. Ce n'est qu'à ces conditions que les professionnels s'impliquent en recherchant de nouvelles pratiques, réseaux et outils. Ici, le patient se doit d'être moteur du projet et les étapes de l'accompagnement sont construites à partir de sa demande. Le chauffeur a dû particulièrement faire preuve de motivation pour que des solutions soient trouvées. Il recontacte son employeur pour en discuter et obtient son soutien : "Ça lui a redonné espoir quand il a vu $M r R$. dans le journal. Il sétait dit: 'pourquoi pas moi' jusque-là, il n'avait pas idée que c'était possible. Donc, ça lui a donné l'idée et comme ce sont des passionnés ces chauffeurs poids-lourd. Et en même temps, c'est un défi pour lui. Du coup, il est revenu en bilan. Et là, on a fait comme un nouveau départ" (Mme B, chargée d'insertion, centre Lamer)

La chargée d'insertion utilise le registre de la passion, comme gage de motivation et d'engagement du travailleur. 
Avec le concours de l'assistante sociale, les professionnels vont aider Rémi à élaborer les étapes d'une reprise du travail qu'ils n'ont jusqu'alors jamais établies.

Il faut en premier lieu valider le permis " poids-lourd " de Rémi, chose très rare avec un tel handicap. Le service, qui n’avait pas de véhicule adapté à cet effet, a fait appel à l'ancien patient, objet de l'article, dont l'employeur avait acquis un véhicule. Après un accord pour le prêt du camion, il a fallu convoquer un agent de l'État pour attester de la capacité de Rémi à conduire le véhicule. Celui-ci a dû repasser deux fois l'examen, avant de finalement réussir. La validation du permis n'est que la première étape du retour à l'emploi.

Se pose ensuite la question du financement du véhicule et de ses adaptations. Ce n'est qu'après une réflexion de plusieurs mois avec une étude de faisabilité et des coûts que l'employeur de Rémi a acquis un camion dédié. Les adaptations ont été réalisées avec l'aide technique du service d'insertion. À l'issue de différentes étapes, Rémi a pris possession d'un nouveau véhicule spécialement adapté à ses difficultés. Ensuite, l'assistante sociale a mis en place un dispositif de reprise progressive du travail, financé en partie par l'entreprise et la sécurité sociale car Rémi, a l'issue des trois ans d'arrêt maladie, n'avait plus droit au temps partiel thérapeutique. L'implication à la fois de l'assistante sociale, du service d'insertion, de l'employeur, de différents financeurs et acteurs, qu'ils soient étatiques ou non (les fournisseurs d'adaptation du véhicule, par exemple) a permis le retour de ce patient au travail.

Les démarches de la chargée d'insertion, tant administratives que pratiques, ont permis d'établir une collaboration durable avec les différents acteurs du projet.

Le cas de Rémi illustre comment, à partir d'un patient, moteur dans son insertion, et de conditions favorables, se construit un accompagnement individualisé. Contrairement aux situations d'accompagnement personnalisé où les propositions sont émises par le service d'insertion, ici, les professionnels doivent construire de nouveaux outils (protocole pour la revalidation du permis poids-lourd, construction d'un réseau de professionnels de fournisseurs de véhicules poids-lourds et des adaptations, financement de véhicules adaptés, mise en place d'un agencement de dispositifs de reprise du travail pour qu'elle soit progressive et assistée, etc.), pour répondre aux besoins et situations inédits du patient. Ce dernier est appréhendé, dans ce cas, comme une personne déjà autonome dans sa capacité de se projeter.

Au final, nous avons examiné deux situations qui mettent en avant, pour l'un, un parcours d'accompagnement connu et maitrisé où le patient a une autonomie relative ; pour l'autre, un parcours inédit et à construire où le patient est considéré comme autonome. Dans tous les cas, l'insertion professionnelle est envisagée dans un cadre particulièrement protecteur. 


\section{Conclusion}

\section{- L'autonomie relative du patient dans un protocole personnalisé}

Dans les services d'insertion sociale et professionnelle au sein des centres hospitaliers de médecine physique et de réadaptation (MPR), les professionnels construisent les objectifs d'insertion du patient selon les fondements de la réadaptation (Barnes \& Ward, 2000). Ils basent leur travail sur des étapes qui constituent des protocoles. Ceux-ci sont reproduits d'un patient à un autre et sont adaptés à la situation de la personne. Le protocole est ainsi préexistant au projet du patient.

Ici, l'autonomie est à la fois un prérequis et un but, elle "sert à la fois de point de référence pour qualifier les personnes et d'objectif à atteindre pour la personne et les soignants" (Winance, 2007). En effet, pour stabiliser un état de santé, le patient doit être autonome fonctionnellement et dans sa vie quotidienne ; le projet professionnel n'est qu'un moyen pour qu'il devienne autonome socialement et économiquement. Pour le patient, l'autonomie, comme pouvoir d'agir, réside dans le fait d'accepter ou non le projet d'insertion proposé. En revanche, cette adhésion est nécessaire comme un contrat moral passé avec le service d'insertion, mais aussi pour permettre l'accès aux réseaux professionnels et sociaux du patient.

L'accompagnement consiste en l'adaptation d'outils standardisés ou de protocoles existants. Il suit des étapes et utilise une palette d'instruments déjà expérimentés qui peuvent être réadaptés en fonction de la personne. Il s'agit dès lors d'une personnalisation de l'accompagnement.

\section{- Le patient nécessairement autonome dans un accompagnement individualisé}

Dans des situations plus inédites, les services d'insertion peuvent toutefois procéder à un accompagnement construit à partir de l'individu. Lorsque le patient se montre très engagé dans un projet qui apparaît réaliste, les professionnels n’hésitent pas à créer les conditions de sa réalisation.

Le patient doit alors satisfaire certains prérequis : un état de santé stabilisé, un soutien social, professionnel et financier fiable et une volonté quasi infaillible. Ce n'est qu'à ces conditions que les professionnels s'engagent dans la construction d'un parcours individualisé où ils s'impliquent en recherchant de nouvelles pratiques, réseaux et outils.

Ici, le patient est au cœur du projet et c'est à partir de sa demande que sont construites les étapes, alors que dans les cas d'accompagnements personnalisés, c'est le service qui est au cœur des propositions. Le patient est ici appréhendé comme une personne déjà autonome dans sa capacité de se projeter. L'accompagnant le guide et intervient comme un partenaire du projet dans ses aspects techniques (judiciaires, ergonomiques, administratifs, etc.) et se révèle être d'un grand soutien moral. 
Cet accompagnement ne peut exister qu’à condition que la personne ait déjà des ressources et un projet défini, jugé comme faisable. Les professionnels contribuent ainsi à les activer.

Cette vision de l'individu au centre de la démarche d'insertion nécessite que le bénéficiaire de l'aide soit proactif et envisage son projet de manière autonome. Les travailleurs sociaux sont des guides qui lui fournissent les outils pour décider et agir.

Ici, comme dans différentes recherches (Astier \& Duvoux, 2006), les dispositifs de prise en charge proposent plus souvent un accompagnement personnalisé utilisant des protocoles déjà maîtrisés, qu'un réel accompagnement individualisé, basé sur le projet et les ressources du patient, plus coûteux en investissement professionnel et personnel.

\section{- Une autonomie de décision dans un dispositif d'insertion professionnelle médico- social protecteur}

Les politiques d'insertion actuelles attendent de leurs bénéficiaires qu'ils s'engagent contractuellement dans un projet, mais aussi qu'ils fassent la preuve de leur implication par des actions concrètes (postuler à des emplois, entreprendre une formation, etc.) (Dang \& Zajdela, 2009 ; Serrano Pascual, 2004). Les services d'insertion du sanitaire n'ont pas cet objectif.

L'insertion sociale est davantage perçue comme un moyen pour le patient de retrouver une autonomie, dans le sens d'une indépendance du système médico-social. Cependant, les politiques françaises, à l'image des politiques européennes dans le champ du handicap, visent à réduire la dépendance à un système social d'assistance (Vielle, Pochet, \& Cassiers, 2006). De plus, la question de l'employabilité des personnes handicapées est mise en avant, alors que ces personnes étaient vues auparavant comme des bénéficiaires légitimes de revenus de substitution liés à leur incapacité de travail. Or, l'employabilité ne peut être appréhendée que dans un contexte social, politique, professionnel et environnemental (Lo \& Ville, 2013), vision partagée par les services de réadaptation. Ainsi, ces professionnels des centres hospitaliers proposent une aide qui engage moralement le patient, sans pour autant en attendre des actions concrètes. Cet accompagnement ne doit pas avoir de répercussions négatives sur les droits sociaux, à l'instar des services d'insertion du champ social qui sont à la fois accompagnateurs et juges dans l'attribution des droits, comme pour le revenu de solidarité active (RSA) (Duvoux, 2009). Aujourd'hui, dans le champ du handicap, il existe encore une séparation entre les instances de décision, de financement et d'accompagnement, ce qui permet des ajustements et des négociations pour obtenir les «meilleurs » compromis pour la personne, comme l'analyse l'a révélé ; alors que dans le champ du social, le cumul des rôles (accompagnement et attribution des droits) enlève une ressource pour la défense des intérêts de l'accompagné. 
Bibliographie

Astier I. (2007), Les nouvelles règles du social, Paris, Puf.

Astier I. \& Duvoux N. (2006), La société biographique : Une injonction à vivre dignement, Paris, L'Harmattan, pp. 15-31.

Astier I. (2010), Sociologie du social et de l'intervention sociale: Domaines et approches (broché), Paris, Armand Colin.

Bacqué M.-H., \& Biewerner C. (2013), L'empowerment, une pratique émancipatrice, Paris, La Découverte.

Barnes M. P., \& Ward, A. B. (2000), Textbook of rehabilitation medecine, Oxford: Oxford University Press.

Becker H. (2002), Les ficelles du métier. Comment conduire sa recherche en sciences sociales, Paris, La Découverte.

Bertaux D. (1997), Les récits de vie, Paris, Nathan.

Bureau M-C. et Rist B. (2013) «Questions de santé et questions de travail : quelles conciliations dans les commissions d'attribution des droits aux personnes handicapées? ", Sociologies pratiques, 1, 26, pp. 85-99

Callon M. (1986), "Éléments pour une sociologie de la traduction. La domestication des coquilles Saint-Jacques et des marins-pêcheurs dans la baie de Saint- Brieuc ", L'année sociologique, 36, pp. 169-208.

Castel R. \& Haroche C. (2001), Propriété privée, propriété sociale, propriété de soi. Entretiens sur la construction de l'individu moderne, Paris, Fayard.

Castra D. (2003), Linsertion professionnelle des publics précaires, Paris, Puf.

Dang A.-T. \& Zajdela H. (2009), "Fondements normatifs des politiques d'activation : un éclairage à partir des théories de la justice ", Recherches économiques de Louvain, 75(3), pp. 313-352.

Demazière D. \& Dubar C. (1997), Analyser les entretiens biographiques. L'exemple de récits d'insertion, Paris, Nathan.

Duvoux N. (2009), L'autonomie des assistés, Paris, Puf.

Ehrenberg A. (2010), La Société du malaise, Paris, Odile Jacob.

Ehrenberg A. (2011), "Souffrir au travail : purger les passions ou ouvrir la voie à l'action?", Esprit (10), pp. 148-157.

Frattini M.-O. (2008), La médecine de rééducation et réadaptation fonctionnelles en France entre médecine et politique. Santé, Population, Politiques sociale, Paris, EHESS, 124 p. 
Hassenteufel P. (2010) «Les processus de mise sur agenda : sélection et construction des problèmes publics ", Informations sociales, 1, 157, pp. 50-58.

Jaeger M. (2009), « Du principe de responsabilité au processus de responsabilisation », Vie sociale, 3, pp. 71-81.

Lascoumes P., Simard L. (2011), «L'action publique au prisme de ses instruments », Revue française de science politique, 61, pp. 5-22.

Lo S.-H. \& Ville I. (2013), "The "employability" of disabled people in France: A labile and speculative notion to be tested against the empirical data from the 2008 "HandicapSanté" study», ALTER, European Journal of Disability Research, 7(4), pp. 227-243.

Méda D. (1995), Le travail : une valeur en voie de disparition, Paris, Aubier.

Paul M. (2007), "Ce qu'accompagner veut dire », Carriérologie, Revue francophone internationale $9(1-2)$.

Ravaud J.-F. (1999), " Modèle individuel, modèle médical, modèle social: la question du sujet ", Handicap, revue de sciences humaines et sociales, 81,pp. 64-75.

Sen A. (1987), Commodities and Capabilities, New Delhi: Oxford India Paperbacks.

Serrano Pascual A. (2004), Les politiques d'employabilité en Europe, Geneva International Labour Office.

Vielle P., Pochet P. \& Cassiers I. (2006), L'État social actif - Vers un changement de paradigme?, Bruxelles : PIE Peter Lang.

Winance M. (2007), « Dépendance versus autonomie... De la signification et de l'imprégnation de ces notions dans les pratiques médicosociales ", Sciences sociales et santé, 25(4), pp. 83-91. 